\title{
Existence de cycles pour des multi-applications du cercle
}

\author{
JEAN-PAUL DUFOUR \\ Gétodim, Institut de Mathématiques, U.S.T.L., Pl. E. Bataillon, \\ 34000 Montpellier, France
}

(Received 30 October 1986 and revised 12 February 1987)

\begin{abstract}
We consider multi-applications $\Gamma$ of the circle $S^{1}$, the graphs of which are 'degree $(1,1)$ ', continuous piece-wise monotonic curves of $S^{1} \times S^{1}$. In general $\Gamma^{p}$ is not a connected curve but it is a union of a degree $(1,1)$ continuous curve $\Gamma_{p}$ of $S^{1} \times S^{1}$ and of some other curves homotopic to a point. Using these $\Gamma_{p}$ we are able to study dynamics of $\Gamma$. We focus on the case where $\Gamma$ has no periodic points and we see, for instance, that all 'regular' orbits have, on $S^{1}$, the same order as orbits of an irrational rotation. Using this we prove that such $\Gamma$ without 'cycles' are obtained from a Denjoy's counter-example, perturbing it in the holes of the invariant set. Finally we generalize the classical result of Block and Franke showing that if $\Gamma$ is a $C^{2}$ curve with no degenerate critical points, or if $\Gamma$ is a $C^{\infty}$ curve with no 'flat' points, there are always 'cycles', unless $\Gamma$ is an homeomorphism.
\end{abstract}

\section{Introduction, résultats principaux}

Le cercle $S^{1}$ est identifié à $\mathbb{R} / \mathbb{Z}$ et on note $\pi: \mathbb{R} \rightarrow S^{1}$ la projection canonique.

Soient $X$ et $Y$ deux ensembles. Une multi-application $\Gamma$ de $X$ dans $Y$ est donnée par son graphe (encore noté $\Gamma$ ) qui est une partie arbitraire de $X \times Y$. Si $\Gamma^{\prime}$ est une multi-application de $Y$ dans $Z, \Gamma^{\prime} \circ \Gamma$ est l'ensemble des $(x, z)$ dans $X \times Z$ tels qu'il existe $y$ dans $Y$ avec $(x, y)$ dans $\Gamma$ et $(y, z)$ dans $\Gamma^{\prime} ; \Gamma^{-1}$ est l'ensemble des $(x, y)$ tels que $(y, x)$ soit dans $\Gamma$; si $X$ est égal à $Y$ on désigne par $\Gamma^{p}$ ( $p$ entier positif) la multi-application obtenue en composant $\Gamma$ avec elle-même $p$ fois; $\Gamma^{-p}$ désigne $\left(\Gamma^{-1}\right)^{p}$.

On notera $P_{1}$ l'ensemble des applications continues $f: \mathbb{R} \rightarrow \mathbb{R}$ telles que

$$
f(x+1)=f(x)+1
$$

pour tout $x$ et qui sont 'monotones par morceaux' en ce sens qu'il existe une partition finie de $[0,1]$ en intervalles sur chacun desquels $f$ est strictement monotone.

Rappelons que toute courbe continue $\gamma: S^{1} \rightarrow S^{1} \times S^{1}$ se relève en une application continue $\bar{\gamma}: \mathbb{R} \rightarrow \mathbb{R} \times \mathbb{R}$ telle que $(\pi \times \pi)(\bar{\gamma}(t))=\gamma(\pi(t))$.

Definition 1. Nous appelons multi-application monotone par morceaux de $S^{1}$ toute multi-application $\Gamma$ de $S^{1}$ dans $S^{1}$ dont le graphe est une courbe $t \rightarrow(\alpha(t), \beta(t)): S^{1} \rightarrow$ $S^{1} \times S^{1}$ admettant un relèvement $t \rightarrow(\bar{\alpha}(t), \bar{\beta}(t)): \mathbb{R} \rightarrow \mathbb{R} \times \mathbb{R}$ où $\bar{\alpha}$ et $\bar{\beta}$ sont dans $P_{1}$.

Comme cas particuliers de telles multi-applications monotones par morceaux, nous trouvons les applications $f$ monotones par morceaux de degré 1 et leurs inverses $f^{-1}$ (au sens des multi-applications). 
Notre but est d'étudier ces multi-applications du point de vue des systèmes dynamiques discrets: on veut étudier le comportement des 'orbites' de $\Gamma$, c'est-à-dire des suites $\left(x_{k}\right)_{k \in Z}$ de points de $S^{1}$ telles que, pour tout $k,\left(x_{k}, x_{k+1}\right)$ soit dans $\Gamma$ et, tout spécialement, l'existence d'orbites périodiques ( $x_{k}$ est égal à $x_{0}$ pour un entier $k$ non nul).

On tombe naturellement sur de telles multi-applications de $S^{1}$ dans différents problèmes. En particulier lorsque l'on étudie un couple $(f, g)$ d'applications différentiables d'une variété compacte $V$ dans $\mathbb{R}$. Génériquement $f$ et $g$ ne sont pas indépendantes le long d'un nombre fini de courbes difféomorphes à $S^{1}$ et il nous faut étudier $(f, g)$ en restriction à ces courbes. Or le comportement de $(f, g)$ en restriction à l'une de ces courbes, notée $S$, est caractérisé par la multi-application $\Gamma \circ \Gamma^{\prime}$ où $\Gamma=\{(x, y) \in S \times S ; f(x)=f(y)\}, \Gamma^{\prime}=\{(x, y) \in S \times S ; g(x)=g(y)\}$.

Essentiellement $\Gamma \circ \Gamma^{\prime}$ est une multi-application monotone par morceaux. Dans [5] nous appliquons les résultats du présent article pour prouver qu'il n'y a pas de couple $C^{\infty}$ stable (au sens de la stabilité des couples, voir [6] et son erratum). Ces multi-applications apparaissent aussi dans le problème classique de Dirichlet hyperbolique où l'on cherche à résoudre l'équation

$$
\partial^{2} u / \partial x \partial y=0
$$

$u: D \rightarrow \mathbb{R}$ est la fonction inconnue, $D$ étant un domaine du plan limité par une courbe fermée $S$ et on impose une condition limite

$$
\left.u\right|_{s}=f
$$

où $f$ est donnée. Lorsque $S$ est convexe le rôle fondamental est joué par l'homéomorphisme $\Gamma: S \rightarrow S$ défini, pour presque tout $x$ dans $S$, en prenant pour $\Gamma(x)$ le point $y$ de $S$ tel qu'il existe $z$ dans $S(z \neq x, z \neq y)$ avec $x$ et $z$ sur la même verticale $z$ et $y$ sur la même horizontale (voir [1], dernier paragraphe, et les références citées là). Lorsque $S$ n'est plus convexe $\Gamma$ devient une multi-application qui est 'génériquement' monotone par morceaux. Signalons enfin que F. Przytycki utilise dans [10] les multi-applications de $S^{1}$ dans l'étude des bifurcations de difféomorphismes (Il a lui aussi, indépendamment, dégagé les notions 'd'âme' et de 'bulle' que nous rencontrerons ci-après).

Lorsque l'on compose deux multi-applications monotones par morceaux on n'obtient pas forcément une multi-application monotone par morceaux. On contourne cette difficulté dans le paragraphe 1: le résultat prouvé là permet de voir que, si $\Gamma$ et $\Gamma^{\prime}$ sont deux multi-applications monotones par morceaux de $S^{1}$, alors $\Gamma \circ \Gamma^{\prime}$ est formée d'un certain nombre de courbes fermées homotopes à un point (que nous appelons bulles de $\Gamma \circ \Gamma^{\prime}$ ) et d'une courbe qui est encore une multiapplication monotone par morceaux de $S^{1}$. Cette courbe, que nous appelons l'âme de $\Gamma \circ \Gamma^{\prime}$, contient l'essentiel des informations concernant $\Gamma \circ \Gamma^{\prime}$. Grâce à cette technique on peut étudier les itérées de $\Gamma$ : en fait on remplace $\Gamma^{k}$ par l'âme de $\Gamma^{k}$, que l'on note $\Gamma_{k}$, et qui est, pour tout $k$, multi-application monotone par morceaux de $S^{1}$.

Soit $f: S^{1} \rightarrow S^{1}$ de classe $C^{1}$; un point critique de $f$ est dit de type pli si c'est un point critique isolé et si la dérivée de $f$ change de signe en ce point. Le théorème 
de Block et Franke ([4]) dit que si $f$ a une dérivée à variation bornée et si elle possède un nombre non nul de points critiques, tous de type pli, alors elle admet des points périodiques. Notre résultat essentiel (Théorème 2 ci-dessus) est une généralisation de ce théorème aux multi-applications de $S^{\mathbf{1}}$.

Definition 2. Soit $\Gamma$ une multi-application monotone par morceaux de $\boldsymbol{S}^{1}$. On dit que le point $(x, y)$ de $S^{1} \times S^{1}$ est un point critique horizontal (resp. vertical) de $\Gamma$ si, pour tout paramétrage $t \rightarrow(\alpha(t), \beta(t))$ de $\Gamma$ tel que dans la définition $1, \alpha($ resp. $\beta)$ admet un extrémum local en ce point. Les points de rebroussements sont ceux qui sont à la fois critique horizontal et vertical. Nous appelons cycle de $\Gamma$ toute famille $\left(x_{0}, x_{1}, \ldots, x_{q-1}\right)$ de points deux à deux distincts de $S^{1}$ vérifiant l'une des propriétés $a, b$ ou c suivantes:

(a) Orbite périodique:

$$
\left(x_{q-1}, x_{0}\right) \in \Gamma ;\left(x_{i}, x_{i+1}\right) \in \Gamma, \quad i=0, \ldots, q-2 ;
$$

avec, comme cas particulier, le cas du point fixe $\left\{x_{0}\right\}$ :

$$
\left(x_{0}, x_{0}\right) \in \Gamma \text {. }
$$

(b) Auto-intersection:

on a $q$ pair $(q=2 p, p \geq 1)$ et

$$
\left(x_{i}, x_{i+2}\right) \in \Gamma, i=0, \ldots, q-3 ;\left(x_{0}, x_{1}\right) \in \Gamma ;\left(x_{q-2}, x_{q-1}\right) \in \Gamma
$$

autrement dit $\left(x_{0}, x_{q-1}\right)$ apparaît de deux façons différentes comme point de $\Gamma^{p}$ : c'est une 'auto-intersection' de $\Gamma^{p}$. Comme cas particulier nous imposons les couples $(x, y)$ correspondants aux auto-intersections de $\Gamma$ : i.e. pour tout paramétrage $t \rightarrow(\alpha(t), \beta(t))$ de $\Gamma$ il existe $t_{1}$ et $t_{2}$ distincts avec

$$
\alpha\left(t_{1}\right)=\alpha\left(t_{2}\right)=x ; \quad \beta\left(t_{1}\right)=\beta\left(t_{2}\right)=y .
$$

(c) Rebroussements:

on a $q \geq 2,\left(x_{0}, x_{1}\right)$ est critique vertical, $\left(x_{q-2}, x_{q-1}\right)$ est critique horizontal et l'on a

$$
\left(x_{i}, x_{i+1}\right) \in \Gamma, \quad i=0, \ldots, q-2 \text {; }
$$

autrement dit $\left(x_{0}, x_{q-1}\right)$ est un point de rebroussement de $\Gamma^{q-1}$.

Remarquons qu'une application (monotone par morceaux, continue) ne peut avoir que des cycles de type (a).

Rappelons qu'un 'contre-exemple de Denjoy' ([11]) est un homéomorphisme $f: S^{1} \rightarrow S^{1}$ respectant l'orientation, à nombre de rotation irrationnel, et qui n'est pas conjugué à une rotation. Pour un tel $f$ il existe un ensemble invariant $K$ dans $S^{1}$ dont le complémentaire est une réunion d'intervalles $I_{i, j}(i \in \mathbb{N}, j \in \mathbb{Z})$, deux à deux disjoints, tels que, pour tous $i$ et $j$,

$$
f\left(I_{i, j}\right)=I_{i, j+1} \text {. }
$$

Après avoir montré dans le paragraphe 3 que l'on peut associer à toute multiapplication $\Gamma$ monotone par morceaux sans cycle un nombre de rotation (comme pour les applications) on montre, au paragraphe 4 , que $\Gamma$ est en fait un contre exemple de Denjoy modifié dans certains des $I_{i, j} \times I_{i, j+1}$. De façon précise on a le résultat suivant. 
THEOREME 1. Soit $\Gamma$ une multi-application monotone par morceaux de $S^{1}$, sans cycle, qui ne soit pas un homéomorphisme. Alors il existe un contre exemple de Denjoy $f: S^{1} \rightarrow S^{1}$, tel que ci-dessus, dont on note $F$ le graphe et il existe une partie finie $A$ de $\mathbb{N} \times \mathbb{Z}$ tel que $\Gamma$ soit obtenue à partir de $F$ en remplaçant $F \cap\left(I_{i, j} \times I_{i, j+1}\right)$ par une courbe $\Gamma_{i, j}$ de même extrémités, incluse dans $I_{i, j} \times I_{i, j+1}$ pour tous $(i, j)$ dans $A$.

Ce théorème donne une généralisation de [2] qui caractérise les applications $f: S^{1} \rightarrow S^{1}$ continues sans points périodiques. On en déduit aisément le théorème qui suit.

ThEOREME 2. Soit $\Gamma$ une multi-application de $S^{1}$ dont le graphe admet un paramétrage $t \rightarrow(\alpha(t), \beta(t)): S^{1} \rightarrow S^{1} \times S^{1}$ où $\alpha$ et $\beta$ sont de classe $C^{1}$, avec des dérivées à variation bornée, n'admettant que des points critiques de type pli et de degré non nul. Alors ou bien $\Gamma$ est un difféomorphisme topologiquement conjugué à une rotation irrationnelle de $S^{1}$ ou bien $\Gamma$ admet des cycles.

Dans le cas particulier où $\Gamma$ est une application $(\alpha=I d)$ on retrouve le théorème de Block-Franke. La même méthode s'applique mot à mot au cas des 'heavy maps' (un cas particulier d'applications non continues) de Misiurewicz ([9]) pour donner un résultat analogue.

Il est possible que l'on puisse raccourcir notre liste de cycles en gardant le même théorème. En tous cas on ne peut la réduire aux seules orbites périodiques: si $h: S^{1} \rightarrow S^{1}$ désigne une application de classe $C^{2}$, de degré 1 , n'admettant que des points critiques de type pli (en nombre non nul) et si $\Delta_{r}$ désigne le graphe de la rotation d'angle irrationnel $r$, alors $(h \times h)^{-1} \Delta_{r}$ contient toujours une courbe $\Gamma_{0}$ qui définit une multi-application de $S^{1}$, vérifiant les hypothèses du théorème, qui n'est pas un difféomorphisme et qui n'a pas d'orbites périodiques (elle a cependant des cycles de type (b) et (c)). Grosso modo ce qui fait marcher le théorème de BlockFranke classique est le fait que, si $f: S^{1} \rightarrow S^{1}$ continue présente une 'oscillation' (3 points qui ont la même image), alors cette oscillation persiste dans toutes les itérées $f^{n}$ et, notant $\bar{f}: \mathbb{P} \rightarrow \mathbb{R}$ un relèvement de $f$, ces oscillations forcent l'un des $\bar{f}^{n}$ à rencontrer l'un des $\Delta_{p}=\{(x, y) / y=x+p\}, p \in \mathbb{Z}$. Le phénomène intéressant qui se produit pour les multi-applications est que (comme dans $\Gamma_{0}$ ci-dessus) une 'oscillation horizontale' peut se combiner avec une 'oscillation verticale' pour former des bulles qui permettent aux relevées $\bar{\Gamma}^{n}$ de 'passer' les $\Delta_{p}, p \in \mathbb{Z}$, sans les rencontrer. Ceci laisse voir que notre résultat ne peut être un corollaire direct des résultats classiques concernant les applications continues (voir [3]).

Une autre voie que nous avions tentée fut de ramener notre étude au cas des applications monotones par morceaux non continues ([8] par exemple): en supprimant des morceaux de $\Gamma$ on trouve une telle application. Outre que ce type de chirurgie comporte un arbitraire discutable, cela ne fonctionne que dans quelques cas particuliers: essentiellement les cas où l'on peut trouver une 'heavy map' ([9]).

Dans un travail en cours de rédaction ([7]) nous donnons une notion adaptée à nos multi-applications 'd'intervalle de rotation'. Evidemment les résultats ne sont pas aussi simples que dans les cas des applications continues ([3]) ou des 'heavy maps'. Cependant Przytycki dans [10] décrit un cas particulier (les 'heavy multifunctions') où l'on a des résultats comparables. 


\section{Deux résultats concernant les applications monotones par morceaux}

Lemme 1. Si $\bar{f}$ et $\bar{g}$ sont dans $P_{1}$ il existe $f^{\prime}$ et $g^{\prime}$ dans $P_{1}$ tels que

$$
\bar{f} \circ f^{\prime}=\overline{\boldsymbol{g}} \circ g^{\prime} \text {. }
$$

De plus si $f^{\prime \prime}$ et $g$ " sont d'autres éléments de $P_{1}$ vérifiant

$$
\bar{f} \circ f^{\prime \prime}=\bar{g} \circ g^{\prime \prime}
$$

alors les courbes $t \rightarrow\left(f^{\prime}(t), g^{\prime}(t)\right)$ et $t \rightarrow\left(f^{\prime \prime}(t), g^{\prime \prime}(t)\right)$ ont au moins un point en commun.

Pour démontrer ce lemme nous aurons besoin des notions qui suivent. Un graphe plongé dans un espace topologique $X$ est une partie $G$ de $X$ formée d'un ensemble discret de points, les 'sommets de $G$ ' et d'une famille de courbes $\left(A_{j}\right)_{j \in J}$, les 'arêtes de $G^{\prime}$, telle que chaque $A_{j}$ soit homéomorphe à $[0,1]$, ses deux extrémités sont des' sommets de $G$ et aucun autre point de $A_{j}$ n'est un sommet. On impose aussi que deux arêtes distinctes ne puissent avoir en commun que des sommets. Un sommet est dit de 'degré $p$ ' $(p \in \mathbb{N})$ s'il est extrémité de $p$ arêtes distinctes. Un 'cycle' de $G$ est déterminé par une suite finie $x_{1}, \ldots, x_{q}$ de sommets de $G$ (répétitions permises) tels que $\left(x_{q}, x_{1}\right),\left(x_{i}, x_{i+1}\right)$ pour $i=1, \ldots, q-1$, soient les extrémités de $q$ arêtes deux à deux distinctes. On peut voir un tel cycle comme un cas particulier de courbe fermée paramétrée par $S^{1}$.

Une application continue $f: S^{1} \rightarrow S^{1}$ est dite de degré $n(n \in \mathbb{Z})$ si elle se relève en une application continue $\bar{f}: \mathbb{R} \rightarrow \mathbb{R}$ telle que $\bar{f}(1)=\bar{f}(0)+n$. Une courbe fermée continue $\gamma: S^{1} \rightarrow S^{1} \times S^{1}$ est dite de degré $(n, p)\left((n, p) \in \mathbb{Z}^{2}\right)$ si ses composantes sont respectivement de degré $n$ et $p$. En particulier une courbe de degré $(0,0)$ est homotope à un point.

On remarque maintenant que l'équation $\bar{f} \circ f^{\prime}=\bar{g} \circ g^{\prime}$ revient à dire que $t \mapsto\left(f^{\prime}(t), g^{\prime}(t)\right)$ est une courbe incluse dans l'ensemble

$$
\bar{G}=\{(\bar{x}, \bar{y}) \in \mathbb{R} \times \mathbb{R} ; \bar{f}(\bar{x})=\bar{g}(\bar{y})\} .
$$

En remarquant qu'il s'identifie à $\bar{g}^{-1} \circ \bar{f}$, nous allons étudier cet ensemble $\bar{G}$.

Raisonnant cas par cas - suivant que $\bar{f}$ (resp. $\bar{g}$ ) est croissante, décroissante, localement maximale ou localement minimale au voisinage de $\bar{x}$ (resp. $\bar{y}$ ) - on obtient pour $\bar{G}$, au voisinage de $(\bar{x}, \bar{y})$ les allures représentées dans la figure 1 qui suit.

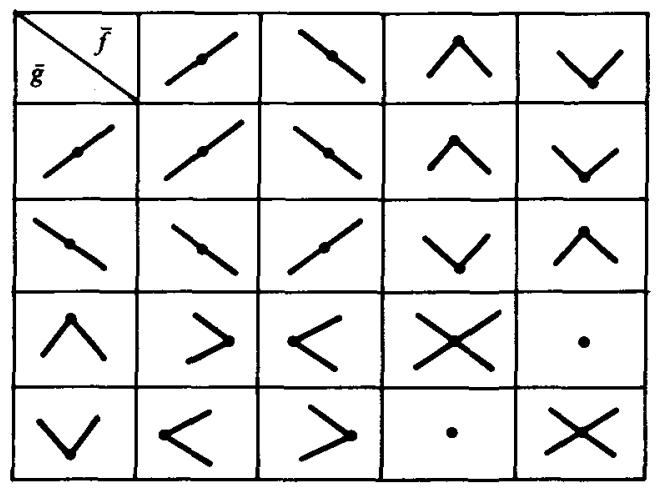

FIGURE 1 
Revenant à la preuve de notre lemme on voit que si $\bar{f}$ (ou $\bar{g}$ ) n'a pas d'extrémum local alors c'est un homéomorphisme et le résultat est trivial. Sinon on fait de $\bar{G}$ un graphe plongé dans $\mathbb{R} \times \mathbb{R}$ de la manière suivante: on prend pour sommets les points $(\bar{x}, \bar{y})$ tels que, ou bien $\bar{x}$ est extrémum local de $\bar{f}$ ou bien $\bar{y}$ est extrémum local de $\bar{g}$; on prend pour arêtes les parties de $\bar{G}$ qui joignent deux sommets.

Nous utiliserons le sous-lemme qui suit.

Sous-Lemme. Soient $f$ et $g$ deux applications continues de $S^{1}$ admettant les relevées respectives $\bar{f}$ et $\bar{g}$ dans $P_{1}$. Alors $G=\left\{(x, y) \in S^{1} \times S^{1} ; f(x)=g(y)\right\}$ est un graphe plongé dans $S^{1} \times S^{1}$ dont tous les sommets sont de degré $0,2,4$ et correspondent à des 'extréma' locaux soit de $f$ soit de $g$ (en dehors du cas où $f$ et $g$ sont toutes deux des homéomorphismes). Si $\gamma$ et $\gamma^{\prime}$ sont deux cycles de ce graphe déterminant des courbes non homotopes à un point, alors ils ont au moins une arête commune. De plus si l'on décompose la partie non discrète de $G$ (i.e. le complémentaire des sommets de degré 0) en une réunion de cycles n'ayant deux à deux en commun que des sommets, alors un et un seul de ces cycles forme une courbe non homotope à un point et c'est une courbe de degré $(1,1)$.

Démonstration du sous-lemme. En dehors du cas trivial où $f$ et $g$ sont des homéomorphismes on fait de $G$ un graphe plongé exactement comme on l'a fait pour $\bar{G}$ : ses sommets sont les points $(x, y)$ où, ou bien $f$ admet un extrémum local, ou bien $g$ admet un extrémum local et l'étude locale prouve qu'ils sont tous de degré $0,2 \mathrm{ou}$ 4 (voir figure 1 ). On a

$$
\pi \times \pi(\bar{G})=G
$$

car, si $(x, y)$ est dans $G$ et $(\bar{x}, \bar{y})$ est dans $(\pi \times \pi)^{-1}(x, y)$, on a

$$
\pi \bar{f}(\bar{x})=\pi \bar{g}(\bar{y})=(f(x)=g(y))
$$

donc on a, pour un certain entier relatif $n$,

$$
\bar{f}(\bar{x})=\bar{g}(\bar{y})+n
$$

d'où l'on tire

$$
\bar{f}(\bar{x})=\bar{g}(\bar{y}+n)
$$

et $(\bar{x}, \bar{y}+n)$ est une préimage de $(x, y)$ par $\pi \times \pi$ qui est dans $\bar{G}$ (l'inclusion réciproque est évidente). On a même plus: toute courbe continue fermée $t \mapsto(x(t), y(t))$ incluse dans $G$ se relève en une courbe incluse dans $\bar{G}$. En effet choisissons un relèvement arbitraire $t \mapsto(\bar{x}(t), \bar{y}(t))$ de cette courbe, on a

$$
\pi \circ \bar{f} \circ \bar{x}(t)=\pi \circ \bar{g} \circ \bar{y}(t)(=f \circ x(t)=g \circ y(t))
$$

pour tout $t$; on en déduit que $\bar{f} \circ \bar{x}$ et $\bar{g} \circ \bar{y}$ sont deux relèvements de la même courbe $(f \circ x)$. On a donc

$$
\bar{f} \circ \bar{x}(t)=\bar{g} \circ \bar{x}(t)+n
$$

pour tout $t$ et un certain entier $n$ fixé. Ainsi on prouve que $t \mapsto(x(t), y(t))$ admet le relèvement $t \mapsto(\bar{x}(t), \bar{y}(t)+n)$ qui est dans $\bar{G}$.

Soit maintenant $\gamma$ un cycle de $G$ non homotope à un point. Un tel cycle admet un paramétrage $t \mapsto(x(t), y(t))$ et se relève, comme ci-dessus, en $\bar{\gamma}: t \mapsto(\bar{x}(t), \bar{y}(t))$ 
qui est dans $\bar{G}$. Supposons que son degré soit $(n, p)$; on a

$$
\bar{f}(\bar{x}(t+1))=\bar{g}(\bar{y}(t+1))=\bar{f}(\bar{x}(t))+n=\bar{g}(\bar{y}(t))+p
$$

d'où l'on tire

$$
n=p \text {. }
$$

En particulier $\bar{x}$ et $\bar{y}$ sont deux applications surjectives de $\mathbb{R}$ dans $\mathbb{R}$. On choisit maintenant un point a de $\mathbb{R}$ qui n'est extrémum local ni de $\bar{f}$ ni de $\bar{g}$ et on considère

$$
\bar{x}_{0}=\inf \{x ; \bar{f}(x)=a\}, \quad \bar{y}_{0}=\inf \{y ; \bar{g}(y)=a\}
$$

Les 'demi-droites' $A=\left\{\left(\bar{x}_{0}, y\right) ; y<\bar{y}_{0}\right\}, B=\left\{\left(x, \bar{y}_{0}\right) ; x<\bar{x}_{0}\right\}$ ne contiennent aucun point de $\bar{G}$; ainsi si $\bar{\gamma}$ ne passait pas par $\left(\bar{x}_{0}, \bar{y}_{0}\right)$ elle serait tout entière dans le quart de plan limité par ce point et les demi-droites $A$ et $B$ ou bien toute entière à l'extérieur de ce quart de plan, cela est incompatible avec ce que nous savons de $\bar{\gamma}$. En conclusion $\bar{\gamma}$ passe par le point $\left(\bar{x}_{0}, \bar{y}_{0}\right)$.

Ceci prouve en particulier que deux cycles non homotopes à un point ont toujours en commun le point $\left(x_{0}, y_{0}\right)=\pi \times \pi\left(\bar{x}_{0}, \bar{y}_{0}\right)$; comme ce point n'est pas un sommet (revoir le choix de a) ces deux cycles ont au moins une arête commune prouvant ainsi la deuxième partie du sous-lemme.

Rappelons que tout graphe fini dont les sommets sont de degré pair non nul se décompose (en général de plusieurs façons) en une réunion de cycles n'ayant en commun deux à deux que des sommets. On décompose ainsi la partie non discrète de G. D'après ce que l'on vient de voir il ne peut y avoir dans cette décomposition qu'un cycle $\gamma$ non homotope à un point. Montrons que s'il existe, ce cycle est de degré $(1,1)$. On a déjà vu qu'il était de degré $(n, n), n \neq 0$; quitte à renverser le paramétrage on peut supposer $\boldsymbol{n}$ positif. Soit, comme plus haut, un paramétrage $t \mapsto(\bar{x}(t), \bar{y}(t))$ de la relevée $\bar{\gamma}$ de $\gamma$. Alors, lorsque $t$ varie de 0 à $1,(\bar{x}(t), \bar{y}(t))$ passe par $\left(\bar{x}_{0}+p, \bar{y}_{0}+p\right), \ldots,\left(\bar{x}_{0}+p+n, \bar{y}_{0}+p+n\right)$ pour un $p$ bien choisi (entier); revenant à $\gamma$ cela voudrait dire qu'il contiendrait $n$ fois l'arête contenant $\left(x_{0}, y_{0}\right)$. On prouve ainsi que $n$ est égal à 1 .

Pour achever la démonstration du sous-lemme il nous reste à voir qu'il existe toujours au moins un cycle de degré $(1,1)$. Si ce n'était pas le cas on n'aurait, dans notre décomposition, que des cycles de degré $(0,0)$ : ils se relèveraient en des courbes fermées de $\mathbb{R}^{2}$. Dans ces conditions $\bar{G}$ serait une réunion de courbes fermées n'ayant deux à deux en commun que des sommets (elle contiendrait toutes les relevées des cycles et rien d'autre car toute arête de $\bar{G}$ se projette en une arête de $G$, qui est dans un cycle). Alors une verticale 'générique' $\bar{x}=C^{t e}$ rencontrerait $\bar{G}$ en un nombre pair de points; ceci contredirait le fait que $\bar{g}^{-1}$ rencontre les verticales génériques en un nombre impair de points (revenir au fait que $\bar{G}$ s'identifie à $\bar{g}^{-1} \circ \bar{f}$ ), achevant ainsi la preuve du sous-lemme.

Revenons maintenant à la preuve du Lemme 1 . En relevant un cycle de degré $(1,1)$ de $G$ on obtient $\bar{y}: t \mapsto(\bar{x}(t), \bar{y}(t)): \mathbb{R} \rightarrow \mathbb{R} \times \mathbb{R}$ qui paramétrise une courbe formée par une réunion d'arêtes de $\bar{G}$. Lorsque $t$ varie de 0 à $1, \bar{\gamma}(t)$ balaye un nombre fini d'arêtes en passant d'un point $(\bar{x}, \bar{y})$ à $(\bar{x}+1, \bar{y}+1)$. Quitte à changer un peu le paramétrage on peut imposer que $\bar{x}$ et $\bar{y}$ soient monotones par morceaux. 
On construira le couple $\left(f^{\prime}, g^{\prime}\right)$ voulu en prenant

$$
f^{\prime}=\bar{x}, \quad g^{\prime}=\bar{y} .
$$

La deuxième partie du Lemme 1 est une paraphrase du fait, déjà vu, que deux telles courbes $t \mapsto(\bar{x}(t), \bar{y}(t))$ passent par l'arête qui contient $\left(\bar{x}_{0}, \bar{y}_{0}\right)$ apparaissant dans la preuve du sous-lemme.

LEMME 2. Soit $f:[a, b] \rightarrow[c, d], g:[e, h] \rightarrow[c, d]$ deux applications continues, strictement monotones par morceaux et telles que

$$
f(a)=c, f(b)=d, g(e)=c, g(h)=d .
$$

Alors il existe deux applications continues, strictement monotones par morceaux $f^{\prime}:[0,1] \rightarrow[a, b], g^{\prime}:[0,1] \rightarrow[e, h]$ avec les relations

$$
\begin{gathered}
f^{\prime}(0)=a, \quad f^{\prime}(1)=b, \quad g^{\prime}(0)=e, \quad g^{\prime}(1)=h, \\
f \circ f^{\prime}=g \circ g^{\prime} .
\end{gathered}
$$

Démonstration. Par des transformations affines on peut se ramener au cas où

$$
[a, b]=[c, d]=[e, h]=[0,1] \text {. }
$$

Alors on peut prolonger $f$ et $g$ de manière évidente en des éléments de $P_{1}$ et on applique le Lemme 1.

\section{Composition de multi-applications}

Soient $\Gamma$ et $\Gamma^{\prime}$ deux multi-applications monotones par morceaux de $S^{1}$ admettant les paramétrages respectifs $t \rightarrow(\alpha(t), \beta(t))$ et $t \rightarrow\left(\alpha^{\prime}(t), \beta^{\prime}(t)\right)$. On note $\bar{\beta}^{\prime}$ et $\bar{\alpha}$ des relevés respectifs de $\beta^{\prime}$ et $\alpha$; on peut les supposer dans $P_{1}$ et, appliquant le Lemme 1 , on peut exhiber $\bar{\alpha}^{\prime \prime}$ et $\bar{\beta}^{\prime \prime}$ dans $P_{1}$ tels que

$$
\bar{\beta}^{\prime} \circ \bar{\alpha}^{\prime \prime}=\bar{\alpha} \circ \bar{\beta}^{\prime \prime} \text {. }
$$

On note $\alpha^{\prime \prime}$ et $\beta^{\prime \prime}$ les projetées respectives de $\bar{\alpha}^{\prime \prime}$ et $\bar{\beta}^{\prime \prime}$.

Definition 3. La multi-application $\Gamma^{\prime \prime}$ de $S^{1}$ admettant le paramétrage $t \rightarrow$ $\left(\alpha^{\prime} \circ \alpha^{\prime \prime}(t), \beta \circ \beta^{\prime \prime}(t)\right)$ est appelée une âme de $\Gamma \circ \Gamma^{\prime}$.

On remarque que l'âme est incluse dans $\Gamma \circ \Gamma^{\prime}$ et que, c'est encore une multiapplication monotone par morceaux de $S^{1}$. Cette âme n'est pas unique mais il résulte de la deuxième partie du Lemme 1 que deux âmes différentes ont forcément un point commun. De plus le sous-lemme du paragraphe 1 montre que le complémentaire de l'âme dans $\Gamma \circ \Gamma^{\prime}$ est réunion d'un nombre fini de courbes fermées homotopes à un point. Ce sont ces courbes que nous appelons des bulles de $\Gamma \circ \Gamma^{\prime}$. Généralisant aux $\Gamma^{k}$ on obtiendra le lemme qui suit.

LEMME 3. Pour tout $k$ dans $\mathbb{Z}$ il existe une multi-application $\Gamma_{k}$ monotone par morceaux de $S^{1}$ telle que $\Gamma_{1}=\Gamma$ et que, pour tous entiers relatifs $p$ et $q$, on ait

$$
\Gamma_{p+q} \subset \Gamma_{p} \circ \Gamma_{q} .
$$

De plus, pour tout $x_{0}$ arbitraire dans $S^{1}$, on peut construire une suite $\left(x_{k}\right)_{k \in Z}$ telle que, pour tous entiers relatifs $p$ et $q$, on ait

$$
\left(x_{q}, x_{p+q}\right) \in \Gamma_{p} .
$$


Vocabulaire. La multi-application $\Gamma_{k}$ sera appelée âme de $\Gamma^{k}$ (elle est incluse dans $\left.\Gamma^{k}\right)$; on notera $t \rightarrow\left(\alpha^{k}(t), \beta^{k}(t)\right)$ un paramétrage de $\Gamma_{k}$. On réservera le nom d'orbite de $\Gamma$ aux suites $\left(x_{k}\right)_{k \in Z}$ données par le Lemme 3.

Par la suite chaque fois que $\gamma$ désigne une application continue de $S^{1}$ dans $S^{1}$ alors $\bar{\gamma}$ désigne une de ses 'relevées' continue de $\mathbb{R}$ dans $\mathbb{R}$.

Démonstration du Lemme 3. Nous écrivons

$$
\alpha_{1}=\alpha, \quad \beta_{1}=\beta \text {. }
$$

D'après le Lemme 1 il existe $\bar{\alpha}_{2}$ et $\bar{\beta}_{12}$ dans $P_{1}$ tels que

$$
\bar{\alpha}_{1} \circ \bar{\beta}_{2}=\bar{\beta}_{1} \circ \bar{\alpha}_{2} \text {. }
$$

De proche en proche on construit, pour tout entier $p$ plus grand que 2 , $\bar{\alpha}_{p}$ et $\bar{\beta}_{p}$ dans $P_{1}$ tels que

$$
\bar{\alpha}_{p-1} \circ \bar{\beta}_{p}=\bar{\beta}_{p-1} \circ \bar{\alpha}_{p}
$$

On définit alors la famille des $\Gamma_{k}$ en posant

$$
\begin{aligned}
\alpha^{0}(t) & =\beta^{0}(t)=t ; \\
\alpha^{k}(t) & =\alpha_{1} \circ \alpha_{2} \circ \cdots \circ \alpha_{k}(t) ; \quad \beta^{k}(t)=\beta_{1} \circ \beta_{2} \circ \cdots \circ \beta_{k}(t)
\end{aligned}
$$

pour $k$ supérieur à 1 et, pour tout $p$ dans $\mathbb{Z}$,

$$
\alpha^{p}(t)=\beta^{-p}(t)
$$

Nous allons prouver (1) dans le cas où $p$ et $q$ sont positifs. Tout point $(x, y)$ de $\Gamma_{p+q}$ est de la forme

$$
x=\alpha_{1} \circ \cdots \circ \alpha_{p+q}(t), \quad y=\beta_{1} \circ \cdots \circ \beta_{p+q}(t) ;
$$

on considère $z=\beta_{1} \circ \cdots \circ \beta_{q} \circ \alpha_{q+1} \circ \cdots \circ \alpha_{q+p}(t)$. Par (3) on arrive à

$$
z=\alpha_{1} \circ \cdots \circ \alpha_{p} \circ \beta_{p+1} \circ \cdots \circ \beta_{q+p}(t) \text {. }
$$

Ceci permet de voir que l'on a à la fois

$$
(x, z) \in \Gamma_{q}, \quad(z, y) \in \Gamma_{p}
$$

et prouve (1) dans ce cas.

Si $p$ et $q$ sont tous deux négatifs (1) se prouve de la même manière en échangeant les rôles des $\alpha$ et de $\beta$. Nous allons prouver (1) dans le cas où $p$ est positif et $q$ négatif $(q=-r)$; le cas où $p$ est négatif et $q$ positif se traite de la même manière. Dans le cas où $p$ est supérieur à $r$ un point $(x, y)$ de $\Gamma_{p+q}$ est de la forme

$$
x=\alpha_{1} \circ \cdots \circ \alpha_{p-r}(t) ; \quad y=\beta_{1} \circ \cdots \circ \beta_{p-r}(t)
$$

utilisant la surjectivité des $\beta_{i}$ on peut exhiber $u$ tel que

et l'on pose

$$
t=\beta_{p-r+1} \circ \cdots \circ \beta_{p}(u)
$$

$$
z=\alpha_{1} \circ \cdots \circ \alpha_{p}(u) .
$$

On a $(x, z)$ dans $\Gamma_{q}$ car, $\operatorname{par}(3)$,

$$
x=\beta_{1} \circ \cdots \circ \beta_{r} \circ \alpha_{r+1} \circ \cdots \circ \alpha_{p}(u)
$$

et $(z, y)$ dans $\Gamma_{p}$ de façon évidente; d'où le résultat dans ce cas. Let cas où $p$ est inférieur à $r$ se traite de manière analogue. 
Montrons maintenant comment construire des orbites $\left(x_{k}\right)_{k \in \mathbb{Z}}$. Par surjectivité de $\alpha_{1}$ on sait, pour tout $x_{0}$ arbitraire donné, trouver $x_{0}^{1}$ tel que

$$
\alpha_{1}\left(x_{0}^{1}\right)=x_{0}
$$

de même on sait trouver $x_{0}^{2}$ tel que

$$
\beta_{2}\left(x_{0}^{2}\right)=x_{0}^{1}
$$

et ainsi on construit pas à pas la suite $\left(x_{0}^{n}\right)_{n \in \mathbb{N}}$ telle que

$$
\beta_{2 i}\left(x_{0}^{2 i}\right)=x_{0}^{2 i-1} ; \quad \alpha_{2 i+1}\left(x_{0}^{2 i+1}\right)=x_{0}^{2 i} .
$$

Alors pour tout $r$ positif on écrit

$$
\begin{aligned}
x_{r} & =\beta_{1} \circ \cdots \circ \beta_{2 r-1}\left(x_{0}^{2 r-1}\right) \\
x_{-r} & =\alpha_{1} \circ \cdots \circ \alpha_{2 r}\left(x_{0}^{2 r}\right) .
\end{aligned}
$$

Alors la relation (2) découle de (3) et du diagramme (commutatif) suivant

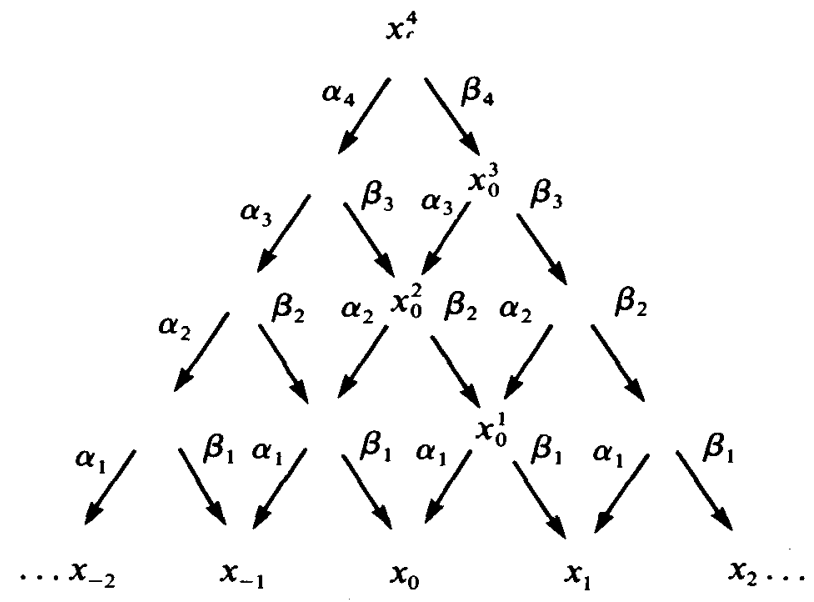

Remarque. La suite des âmes $\left(\Gamma_{k}\right)_{k}$ n'est pas unique mais il nous semble que si $\left(\Gamma_{k}^{\prime}\right)_{k}$ est une autre telle suite on peut affirmer que l'on a pour tout $k$

$$
\bar{\Gamma}_{k} \cap \bar{\Gamma}_{k}^{\prime}=\varnothing \text {, }
$$

en notant $\bar{\Gamma}_{k}$ et $\bar{\Gamma}_{k}^{\prime}$ des relevées de $\Gamma_{k}$ et $\Gamma_{k}^{\prime}$.

\section{Nombre de rotation de multi-applications sans orbites périodiques}

Les notations sont celles du paragraphe 2 . Remarquons qu'en travaillant avec les $\bar{\alpha}_{i}$ et $\bar{\beta}_{i}$ à la place des $\alpha_{i}$ et $\beta_{i}$ on construit, comme dans la preuve du Lemme 3, des suites $\left(\bar{x}_{k}\right)_{k \in \mathcal{Z}}$ de points de $\mathbb{R}$ tels que les $x_{k}=\pi\left(\bar{x}_{k}\right)$ soient les points des orbites de $\Gamma$.

Lemme 4. Si Г n'a pas d'orbite périodique il existe un irrationnel $r$ tel que

$$
\lim _{q \rightarrow \pm \infty} \frac{1}{q} \bar{x}_{q}=r
$$

pour toute $\left(\bar{x}_{k}\right)_{k \in \mathbb{Z}}$ telle que ci-dessus. 
Démonstration. A cause de l'hypothèse le graphe de $\bar{\Gamma}^{k}(k \neq 0)$ ne rencontre aucune des parallèles à la première bissectrice de $\mathbb{R}^{2}$ d'équation $y=x+n, n$ dans $\mathbb{Z}$. Comme l'on a $\bar{\Gamma}_{k}$ inclus dans $\bar{\Gamma}^{k}$, on en déduit l'existence d'une suite d'entiers relatifs $\left(n_{k}\right)_{k \in \mathbf{Z}^{*}}$ tels que

$$
\bar{\Gamma}_{k} \subset\left\{(x, y) ; x+n_{k}<y<x+n_{k}+1\right\} .
$$

Ainsi si $(y, z)$ est dans $\bar{\Gamma}_{k}$ on a, pour $k$ strictement positif,

$$
\left.\frac{z-y}{k} \in \sigma_{k}=\right] \frac{n_{k}}{k}, \frac{n_{k}+1}{k}[\text {. }
$$

Si $\left(x_{k}\right)_{k}$ est une orbite telle que ci-dessus on a, pour $l$ positif,

$$
\bar{x}_{k l}-\bar{x}_{0}=\left(\bar{x}_{k}-\bar{x}_{0}\right)+\left(\bar{x}_{2 k}-\bar{x}_{k}\right)+\cdots+\left(\bar{x}_{k l}-\bar{x}_{k(l-1)}\right)
$$

donc

$$
\frac{\bar{x}_{k l}-\bar{x}_{0}}{k l}=\sum_{i=0}^{l-1} \frac{z^{i}-y^{i}}{k l}=\frac{1}{l} \sum_{i=0}^{l-1} \frac{z^{i}-y^{i}}{k}
$$

avec

$$
y^{i}=\bar{x}_{k i}, \quad z^{i}=\bar{x}_{k(i+1)} ;
$$

or (1) implique, pour tout $i$,

$$
\left(\bar{x}_{k i}, \bar{x}_{k(i+1)}\right) \in \bar{\Gamma}_{k} .
$$

On en déduit

$$
\frac{\bar{x}_{k l}-\bar{x}_{0}}{k l} \in \sigma_{k} .
$$

En échangeant les rôles de $k$ et $l$ on a

$$
\frac{\bar{x}_{k l}-\bar{x}_{0}}{k l} \in \sigma_{1} .
$$

Donc les $\sigma_{k}, k>0$, se rencontrent deux à deux. Comme leurs diamètres tendent vers 0 avec $k$ on en déduit

On en déduit, utilisant

$$
\bigcap_{k} \bar{\sigma}_{k}=\{r\}
$$

$$
\frac{\bar{x}_{k}-\bar{x}_{0}}{k} \in \sigma_{k}
$$

que $\bar{x}_{k / k}$ tend vers $r$ lorsque $k$ tend vers $+\infty$. On en déduit aussi que $n_{k} / k$ tend vers $r$ dans les mêmes conditions. Comme $\bar{\Gamma}_{-k}$ est le symétrique de $\bar{\Gamma}_{k}$ par rapport à la première bissectrice on voit que $n_{-k} /-k\left(=n_{k}+1 / k\right)$ a encore $r$ pour limite et on en déduit que $\bar{x}_{k} / k$ tend vers $r$ lorsque $k$ tend vers $-\infty$.

Supposons maintenant que $r$ soit égal au rationnel $p / q$; dans le cas $p \leq n_{q}$ on peut affirmer l'existence d'un nombre $\epsilon$ strictement positif avec

$$
y>x+p+\varepsilon
$$

pour tout $(x, y)$ dans $\Gamma_{q}$ et l'on a

$$
\frac{\bar{x}_{q l}-\bar{x}_{0}}{q l}=\frac{1}{q l} \sum_{i=0}^{l-1}\left(\bar{x}_{q(i+1)}-\bar{x}_{q i}\right)>r+\varepsilon / q,
$$


ce qui est incompatible avec le fait que le premier membre tende vers $r$ lorsque $l$ tend vers l'infini. On raisonne de manière analogue dans le cas $p \geq n_{q}+1$ pour prouver que $r$ ne peut être rationnel, achevant ainsi la preuve.

Vocabulaire. Ce nombre $r$ sera appelé nombre de rotation de $\Gamma$.

Cette notion généralise à ce cas la notion classique de nombre de rotation d'un homéomorphisme du cercle. On remarque que le nombre $r$ construit ci-dessus dépend à priori de la suite d'âmes $\left(\Gamma_{k}\right)_{k}$ choisie: on peut conjecturer qu'il ne dépend que de $\Gamma \backslash$ (à un entier près); un des corollaires du théorème 1 est que c'est le cas si $\Gamma$ n'a pas de cycles.

Lемме 5. Si $\Gamma$ n'a pas d'orbite périodique et admet le nombre de rotation $r$ les orbites de $\Gamma$ sont rangées sur $S^{1}$ comme les orbites de la rotation (irrationnelle) d'angle $r$.

Démonstration. Il nous suffit de comparer les ordres des points $x_{i}, x_{i+p}, x_{i+p+m}$ (avec $m$ et $p$ strictement positifs) avec ceux de $y_{i}, y_{i+p}, y_{i+p+m}$ où $\left(y_{k}\right)_{k \in Z}$ est une orbite de la rotation d'angle $r$. Revenant à $\mathbb{R}$ cela nous mène à la comparaison de l'ordre des couples $(a, b)$ et $\left(a^{\prime}, b^{\prime}\right)$ où

$$
\begin{aligned}
a & =\bar{x}_{i+p}-\bar{x}_{i}-\left[\bar{x}_{i+p}-\bar{x}_{i}\right], \\
b & =\bar{x}_{i+p+m}-\bar{x}_{i}-\left[\bar{x}_{i+p+m}-\bar{x}_{i}\right], \\
a^{\prime} & =\bar{y}_{i+p}-\bar{y}_{i}-\left[\bar{y}_{i+p}-\bar{y}_{i}\right]=p r-[p r], \\
b^{\prime} & =\bar{y}_{i+p+m}-\bar{y}_{i}-\left[\bar{y}_{i+p+m}-\bar{y}_{i}\right]=(p+m) r-[(p+m) r],
\end{aligned}
$$

et $[x]$ désigne la partie entière de $x$.

On remarque maintenant que pour tout $k$ positif on a

$$
\left[\bar{x}_{k+i}-\bar{x}_{i}\right]=[k r]=n_{k} \text {. }
$$

Cela provient des deux relations

$$
n_{k}<\bar{x}_{k+i}-\bar{x}_{i}<n_{k}+1, \quad n_{k} / k<r<n_{k}+1 / k
$$

Alors on a les équivalences

$$
\begin{aligned}
a<b & \Leftrightarrow \bar{x}_{i+p}-\bar{x}_{i}-n_{p}<\bar{x}_{i+p+m}-\bar{x}_{i}-n_{p+m} \\
& \Leftrightarrow n_{p+m}-n_{p}<\bar{x}_{i+p+m}-\bar{x}_{i+p} \Leftrightarrow n_{p+m}-n_{p} \leq n_{m} \\
& \Leftrightarrow[(p+m) r]-[p r]<(p+m) r-p r \Leftrightarrow p r-[p r]<(p+m) r-[(p+m) r] \\
& \Leftrightarrow a^{\prime}<b^{\prime} .
\end{aligned}
$$

On en déduit le résultat.

Lemme 6. On note $\Gamma^{\prime}$ la multi-application de $S^{1}$ paramétrée par $t \rightarrow\left(\alpha_{2}(t), \beta_{2}(t)\right)$ (notation du paragraphe 2). Si $\Gamma$ n'admet pas d'orbite périodique et a le nombre de rotation $r$ il en est de même de $\Gamma^{\prime}$.

Démonstration. Pour tout $k$ plus grand que 2 on pose

$$
\begin{aligned}
& \alpha^{k^{\prime}}(t)=\alpha_{2} \circ \alpha_{3} \circ \cdots \circ \alpha_{k+1}(t), \\
& \beta^{k^{\prime}}(t)=\beta_{2} \circ \beta_{3} \circ \cdots \circ \beta_{k+1}(t) ;
\end{aligned}
$$

cela définit une suite d'âmes $\left(\Gamma_{k}^{\prime}\right)_{k \in Z}$ pour $\Gamma^{\prime}$ en prenant pour $\Gamma_{k}^{\prime}(k>0)$ la courbe 
$t \rightarrow\left(\alpha^{k^{\prime}}(t), \beta^{k^{\prime}}(t)\right)$. On a les diagrammes commutatifs

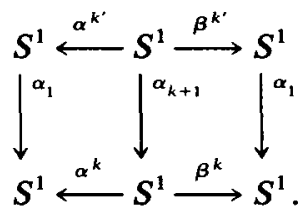

Alors $\bar{\alpha}_{1} \times \bar{\alpha}_{1}$ est une application de $\mathbb{R} \times \mathbb{R}$ dans lui-même qui envoie $\bar{\Gamma}_{k}^{\prime}$ sur $\bar{\Gamma}_{k}, \bar{\Gamma}^{\prime k}$ sur $\bar{\Gamma}^{k}$ et qui conserve les parallèles à la première bissectrice d'équations $y=x+n, n$ dans $\mathbb{Z}$. On en déduit que si $\bar{\Gamma}^{\prime k}$ rencontrait l'une de ces droites il en serait de même de $\bar{\Gamma}^{k}$ ce qui n'est pas; donc $\Gamma^{\prime}$ n'a pas de points périodiques.

On note $\left(\boldsymbol{n}_{k}^{\prime}\right)_{k \in \mathbf{Z}^{*}}$ la famille d'entiers relatifs tels que

$$
\bar{\Gamma}_{k}^{\prime} \subset\left\{(x, y) ; x+n_{k}^{\prime}<y<x+n_{k}^{\prime}+1\right\} .
$$

Notons, pour tout entier relatif $n$, par $D_{n}$ la portion du plan $\mathbb{R} \times \mathbb{R}$ comprise entre les droites $y=x+n$ et $y=x+n+1$. Comme $\bar{\alpha}_{1}$ est bornée sur tout intervalle de longueur 1 il existe un entier $m$ (indépendant de $n$ ) tel que

On en déduit

$$
\bar{\alpha}_{1} \times \bar{\alpha}_{1}\left(D_{n}\right) \subset \bigcup_{n-m<j<n+m} D_{j} .
$$

$$
n_{k}^{\prime}-m \leq n_{k} \leq n_{k}^{\prime}+m
$$

et donc

$$
\lim _{k \rightarrow \infty} n_{k}^{\prime} / k=\lim _{k \rightarrow \infty} n_{k} / k
$$

Ceci achève la preuve de notre lemme.

\section{Etude des multi-applications de $S^{1}$ sans cycles}

Ce paragraphe est consacré à la démonstration du Théorème 1. Les notations sont celles des deux paragraphes précédents. Nous faisons l'hypothèse que $\Gamma$ n'est pas un homéomorphisme et n'a pas de cycle.

On suppose le paramétrage $t \rightarrow\left(\alpha_{1}(t), \beta_{1}(t)\right)$ choisi de telle façon que $\alpha_{1}$ ait le minimum possible d'extréma locaux.

On choisit une fois pour toute une suite $\left(\bar{x}_{k}\right)_{k \in Z}$ construite comme dans le paragraphe précédent et dont la projetée $\left(x_{k}\right)_{k \in Z}$ est une orbite de $\Gamma$. D'après le Lemme $5,\left(x_{k}\right)_{k \in Z}$ est rangée sur $S^{1}$ comme les orbites pour la rotation d'angle irrationnel $r$ (où $r$ est le nombre de rotation de $\Gamma$ ). On note $K$ l'ensemble des points d'accumulation de $\left(x_{k}\right)_{k \in \mathbf{Z}}$.

Remarquons d'abord que, si $K$ est égal à $S^{1}$, alors on peut construire un homéomorphisme $h$ de $S^{1}$ dans $S^{1}$ en imposant, pour une orbite $\left(y_{k}\right)_{k \in Z}$ de la rotation d'angle $r$, pour tout $k$,

$$
h\left(y_{k}\right)=x_{k}
$$

et en complétant par continuité. L'homéomorphisme $h \times h$ envoie le graphe $\boldsymbol{R}_{r}$ de la rotation sur une partie de $\Gamma$. Comme $\Gamma$ ne peut se restreindre à cette partie ( $\Gamma$ n'est pas un homéomorphisme) on en déduit l'existence de points multiples pour $\Gamma$ donc de cycles du type $b$ (Définition 2 ). 
Pour la suite nous supposerons donc que $K$ est différent de $S^{1}$ : il existe des arcs de $S^{1}$ ne contenant aucun point de $K$.

Soit $x$ un point de $S^{1}$. Comme $K$ n'a pas de point isolé, il existe deux points $\vec{x}$ et $\bar{x}$ de $K$ et deux sous-suites $\left(x_{\varphi(n)}\right)_{n},\left(x_{\psi(n)}\right)_{n}$ de $\left(x_{k}\right)_{k}$, respectivement strictement croissantes et décroissantes (en un sens évident) telle que

$$
\lim _{n} x_{\varphi(n)}=\vec{x}, \quad \lim _{n} x_{\psi(n)}=\dot{x},
$$

$\varphi$ et $\psi$ sont croissantes et l'arc $X_{0}$ de $S^{1}$ limité par $\vec{x}$ et $\dot{x}$ contenant $x$ ne contient pas d'autres points de $K$ que $\overrightarrow{\boldsymbol{x}}$ et $\dot{\boldsymbol{x}}$.

Les suites $\left(x_{\varphi(n)+i}\right)_{n}$ et $\left(x_{\psi(n)+i}\right)_{n}$ sont encore respectivement croissantes et décroissantes pour tout $i$ fixé; on note $\vec{x}_{i}$ et $\tilde{x}_{i}$ leurs limites respectives. Notant $X_{i}$ l'arc de $S^{1}$ limité par ces deux points et qui ne contient pas d'autres points de $K$, on construit une suite $\left(X_{k}\right)_{k \in Z}$ d'arcs de $S^{1}$ dont certains peuvent être réduits à un seul point.

Lemme 7. Pour tout $x,\left(\vec{x}_{k}\right)_{k}$ est une orbite de $\Gamma$; en particulier il existe, pour tout entier $j>0$ des suites $\left(\vec{x}_{k}^{j}\right)_{k \in Z}$ de points de $S^{1}$, rangées sur $S^{1}$ comme les points d'une orbite pour la rotation $d$ 'angle $r$ telles que

$$
\begin{array}{ll}
\alpha_{2 i+1}\left(\vec{x}_{k}^{2 i+1}\right)=\vec{x}_{k}^{2 i}, & \alpha_{2 i}\left(\vec{x}_{k}^{2 i}\right)=\vec{x}_{k-1}^{2 i-1} \\
\beta_{2 i+1}\left(\vec{x}_{k}^{2 i+1}\right)=\vec{x}_{k+1}^{2 i}, & \beta_{2 i}\left(\vec{x}_{k}^{2 i}\right)=\vec{x}_{k}^{2 i-1}
\end{array}
$$

pour tout $i$ et tout $k$. De plus pour tout $j>0$ et tout $k$ il existe $\varepsilon>0$ tel que $\beta_{j}$ et $\alpha_{j}$ soient croissantes en restriction $\dot{a}\left[\vec{x}_{k}^{j}-\varepsilon, \vec{x}_{k}^{j}\right]$. On a un résultat analogue en remplaçant $\rightarrow$ par $\leftarrow e t\left[\vec{x}_{k}^{j}-\varepsilon, \vec{x}_{k}^{j}\right]$ par $\left[\vec{x}_{k}^{j}, \vec{x}_{k}^{j}+\varepsilon\right]$.

Démonstration. On suppose que l'orbite $\left(x_{k}\right)_{k}$ est construite comme dans la démonstration du Lemme 3: en particulier il existe une suite $\left(x_{k}^{1}\right)_{k \in Z}$ telle que, pour tout $k$,

$$
\alpha_{1}\left(x_{k}^{1}\right)=x_{k}, \quad \beta_{1}\left(x_{k}^{1}\right)=x_{k+1} .
$$

De plus $\left(x_{k}^{1}\right)_{k \in \mathbf{Z}}$ est une orbite pour $\Gamma^{\prime}$ avec les notations du Lemme 6. Ce Lemme 6 affirme que les $x_{k}^{1}$ sont rangées sur $S^{1}$ comme les $x_{k}$. En particulier $\left(x_{\varphi(n)+k}^{1}\right)_{n}$ est une suite croissante admettant une limite $\vec{x}_{k}^{1}$ et, par continuité,

$$
\alpha_{1}\left(\vec{x}_{k}^{1}\right)=\vec{x}_{k} ; \quad \beta_{1}\left(\vec{x}_{k}^{1}\right)=\vec{x}_{k+1} .
$$

De façon plus générale il existe des suites $\left(x_{k}^{i}\right)_{k}$ avec,

$$
\begin{array}{ll}
\alpha_{2 i+1}\left(x_{k}^{2 i+1}\right)=x_{k}^{i}, & \alpha_{2 i}\left(x_{k}^{2 i}\right)=x_{k}^{2 i-1}, \\
\beta_{2 i+1}\left(x_{k}^{2 i+1}\right)=x_{k+1}^{2 i}, & \beta_{2 i}\left(x_{k}^{2 i}\right)=x_{k}^{2 i-1},
\end{array}
$$

et, itérant la méthode précédente, on construit pas à pas les $\left(\vec{x}_{k}^{t}\right)$ pour $k$ dans $\mathbb{Z}, i>0$, de façon que l'on ait les formules souhaitées. Ceci montre que $\left(\vec{x}_{k}\right)_{k}$ est une orbite construite comme dans la preuve du Lemme 3.

Rappelons nous maintenant que $\bar{\alpha}_{j}$ et $\bar{\beta}_{j}$ sont monotones par morceaux donc elles sont forcément croissantes sur les intervalles $\left[\bar{x}_{\varphi(n)+k}^{j}, \bar{x}_{\varphi(n+1)+k}^{j}\right]$ sauf peut être sur un nombre fini. On en déduit que $\alpha_{j}$ et $\beta_{j}$ sont croissantes au moins sur un petit arc limité à droite par $\vec{x}_{k}^{j}$. Notre résultat en découle.

Par la suite nous raisonnerons par l'absurde en montrant que si l'on n'avait pas certaines propriétés alors $\Gamma$ aurait des points périodiques: dans cet optique le 
Lemme 8 qui suit nous donne une technique pour faire apparaître de tels points. Mais nous donnons avant un peu de vocabulaire.

Soient $I=[a, b]$ et $J=[c, d]$ deux intervalles compacts de $\mathbb{R}$. On dira que $G$ est une multi-application monotone par morceaux de $I$ dans $J$ si son graphe est une courbe paramétrée $t \rightarrow(\alpha(t), \beta(t)), t \in[0,1]$, incluse dans $I \times J$ avec

$$
\alpha(0)=a, \beta(0)=c, \alpha(1)=b, \beta(1)=d
$$

et où $\alpha$ et $\beta$ sont continues et strictement monotones par morceaux.

Lemme 8. Soient $I_{0}, \ldots, I_{n}$ des intervalles compacts de $\mathbb{R}$ et $G_{1}, G_{2}, \ldots, G_{n}$ des multi-applications monotones par morceaux respectivement de $I_{0}$ dans $I_{1}$, de $I_{1}$ dans $I_{2}, \ldots$, de $I_{n-1}$ dans $I_{n}$. Soient deux intervalles compacts $I$ et $K$ de $\mathbb{R}$ tels que

$$
I \supset I_{n}, \quad I_{0} \supset K
$$

et $F$ un homéomorphisme de I sur K. Alors $G_{n} \circ G_{n-1} \circ \cdots \circ G_{1} \circ F$ admet un point fixe.

Démonstration. On peut construire une 'âme' pour $G_{2} \circ G_{1}$ exactement comme on l'a fait pour la composition de deux multi-applications monotones par morceaux de $S^{1}$ en utilisant le Lemme 2 à la place du Lemme 1 (Si $t \mapsto\left(\alpha_{1}(t), \beta_{1}(t)\right)$ et $t \mapsto\left(\alpha_{2}(t), \beta_{2}(t)\right)$ sont de bons paramétrages respectifs de $G_{1}$ et $G_{2}$, le Lemme 2 affirme l'existence de $\alpha$ et $\beta$ convenables tels que $\alpha_{2} \circ \beta=\beta_{1} \circ \alpha$; on prend pour âme la courbe qui admet le paramétrage $\left.t \mapsto\left(\alpha_{1} \circ \alpha(t), \beta_{2} \circ \beta(t)\right)\right)$. On obtient ainsi une multi-application monotone par morceaux de $I_{0}$ dans $I_{2}$ incluse dans $G_{2} \circ G_{1}$. Itérant cette méthode on construit une multi-application $G$ monotone par morceaux de $I_{0}$ dans $I_{n}$ et qui est incluse dans $G_{n} \circ \cdots \circ G_{1}$. Il reste à voir que $G \circ F$ admet un point fixe.

Pour cela on suppose que $K$ et $I$ sont respectivement les intervalles $[a, b]$ et $[c, d]$. Pour des raisons de connexité $G$ contient une partie $G^{\prime}$ qui est une courbe connexe joignant un point $(a, y)$ à un point $(b, z)$ tout en restant dans $[a, b] \times \mathbb{R}$. La composée de $F$ avec $G^{\prime}$ donne une courbe qui joint un point de $\{c\} \times I_{n}$ à un point de $\{d\} \times I_{n}$; pour des raisons de connexité cette courbe doit rencontrer la diagonale principale de $\mathbb{R}^{2}$ donnant ainsi le point fixe cherché.

Vocabulaire. On appelle boîte tout ensemble $X_{k} \times X_{k+1}$ (notion introduite juste avant le Lemme 7). On appelle $X_{k}^{j}$ l'arc limité par $\vec{x}_{k}^{j}$ et $\bar{x}_{k}^{j}$ et ne contenant pas d'autre points $d$ 'accumulation de $\left(x_{k}^{j}\right)_{k}$ (cet arc peut se réduire à un unique point). On dit que $X_{k} \times X_{k+1}$ est une boîte régulière si $X_{k}$ et $X_{k+1}$ ne se réduisent pas à un unique point et si $\Gamma$ définit, en restriction à cette boîte une multi-application monotone par morceaux de $X_{k}$ dans $X_{k+1}$ (de façon précise cela veut dire que l'on a

$$
\left.\alpha_{1}\left(X_{k}^{1}\right)=X_{k}, \quad \beta_{1}\left(X_{k}^{1}\right)=X_{k+1}\right) .
$$

LeMME 9. Si pour tout $k$ strictement supérieur à $0, X_{k} \times X_{k+1}$ est régulière alors $\Gamma$ ne peut contenir aucun arc ayant son origine en un point de $\left\{\vec{x}_{0}, \bar{x}_{0}\right\} \times X_{1}$ et tout entier inclus dans $\left(S^{1}-\dot{X}_{0}\right) \times X_{1}(\AA=$ intérieur de $A)$. Si pour tout $k$ strictement inférieur $\grave{a}$ $0, X_{k-1} \times X_{k}$ est régulière alors $\Gamma$ ne peut contenir aucun arc ayant son origine en un point de $X_{-1} \times\left\{\vec{x}_{0}, \bar{x}_{0}\right\}$ et tout entier inclus dans $X_{-1} \times\left(S^{1}-\dot{X}_{0}\right)$. 
Démonstration. Nous démontrons la première partie (la seconde est analogue) et, pour cela nous raisonnons par l'absurde en supposant l'existence d'un arc $\gamma$ de $\Gamma$ ayant son origine sur $\bar{x}_{0} \times X_{1}$ et tout entier inclus dans $\left(S^{1}-\dot{X}_{0}\right) \times X_{1}$ comme sur la figure 2 qui suit. Cet arc $\gamma$ se projette sur l'axe des $x$ ' en un arc $I$ d'origine $\bar{x}_{0}$ et extérieur à $X_{0}$. En particulier, pour $m$ assez grand $I$ contient $x_{\psi(m)}$.

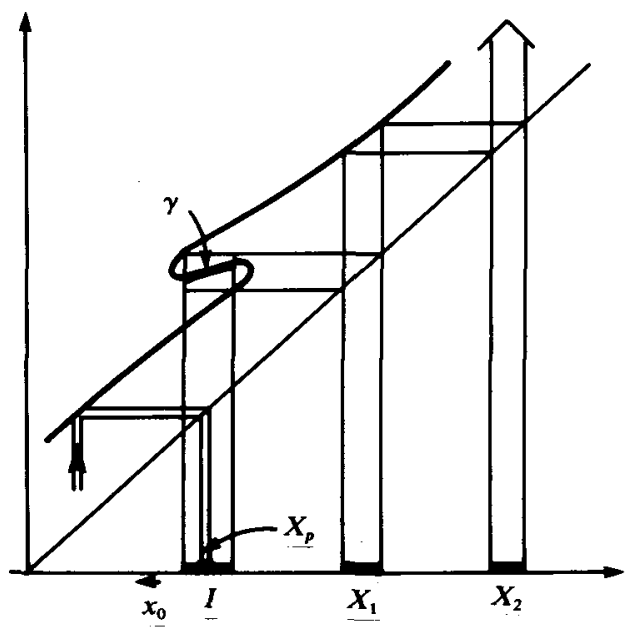

FIGURE 2

On note maintenant que si $\left(z_{k}\right)_{k \in Z}$ est une orbite pour la rotation $R_{r}$ de $S^{1}$ on a $\lim _{n} z_{\psi(n)}=\lim _{n} z_{\varphi(n)}=z$ pour un $z$ bien choisi; pour tout $m$ fixé il existe $p$ tel que $\left(\boldsymbol{R}_{r}\right)^{p}(z)$ soit dans l'arc ouvert $A$ limité par $z_{\psi(m+1)}$ et $z_{\psi(m-1)}$ contenant $z_{\psi(m)}$; comme $\left(R_{r}\right)^{p}(z)$ est limite des suites $q \rightarrow z_{\varphi(q)+p}$ et $q \rightarrow z_{\psi(q)+p}$ il existe $q_{0}$ tel que pour $q>q_{0}$ on ait $z_{\varphi(q)+p}$ et $z_{\psi(q)+p}$ dans $A$. Transportant cette propriété, portant sur l'ordre des $z_{k}$, on en déduit que, pour $q>q_{0}, x_{\varphi(q)+p}$ et $x_{\psi(q)+p}$ sont dans l'arc $B$ limité par $x_{\psi(m+1)}$ et $x_{\psi(m-1)}$ contenant $x_{\psi(m)}$. On a alors

$$
\vec{x}_{p} \in B, \dot{x}_{p} \in B \text {. }
$$

Comme $B$ est inclus dans $I$ pour $m$ assez grand on en déduit

$$
X_{p} \subset I \text {. }
$$

Quitte à restreindre $I, \gamma$ détermine un homéomorphisme $F$ de $I$ sur un intervalle $K$ de $X_{1}$. L'hypothèse de régularité permet de voir que $\Gamma$, convenablement restreint, détermine une famille $G_{1}, G_{2}, \ldots, G_{p-1}$ de multi-applications monotones par morceaux respectivement de $X_{1}$ dans $X_{2}$, de $X_{2}$ dans $X_{3}, \ldots$, de $X_{p-1}$ dans $X_{p}$. Le Lemme 8 nous permet de voir que $G_{p-1} \circ \ldots \circ G_{1} \circ F$ a un point fixe. Ceci est impossible car un tel point serait un point périodique (de 'période' $p$ ) de $\Gamma$. On raisonne de même dans les autres cas

LEMME 10. Ou bien tous les $X_{k}$ sont réduits à un point, ou bien aucun des $X_{k} n$ 'est réduit à un point. 
Démonstration. Supposons que $X_{k}$ et $X_{k+n}(n>1)$ soient réduits à un point alors que $X_{k+1}, \ldots, X_{k+n-1}$ ne le sont pas: dans ce cas

$$
\left(\vec{x}_{k}, \vec{x}_{k+1}, \dot{x}_{k+1}, \ldots, \vec{x}_{k+n-1}, \tilde{x}_{k+n-1}, \vec{x}_{k+n}\right)
$$

est un cycle de type $b$ (Définition 2) ce qui est impossible. Donc, si certains des $X_{k}$ ne se réduisent pas à un seul point on ne peut avoir que les trois cas:

(i) $X_{k}$ ne se réduit à un point pour aucun indice $k$.

(ii) $X_{k}$ ne se réduit pas à un point pour $k>k_{0}$ et $X_{k_{0}}$ se réduit à un point.

(iii) $X_{k}$ ne se réduit pas à un point pour $k<k_{0}$ et $X_{k_{0}}$ se réduit à un point.

Il nous reste à prouver que l'on ne peut avoir le cas (ii) (on démontre de manière symétrique que l'on ne peut avoir (iii)). Pour simplifier les notations nous imposons $k_{0}=0$, ce qui ne change rien.

Notons d'abord que, pour tout $k$ supérieur ou égal à un certain entier positif $\boldsymbol{n}$, toutes les boîtes $X_{k} \times X_{k+1}$ sont régulières et, même, $\Gamma$ en restriction à ces boîtes est un homéomorphisme; en effet si cela n'était pas $\alpha_{1}$ ou $\beta_{1}$ auraient une infinité de zones 'décroissantes', contredisant la propriété d'avoir des relèvement dans $P_{1}$.

D'après le Lemme $7,\left(\vec{x}_{k n}, \vec{x}_{(k+1) n}\right)$ et $\left(\bar{x}_{k n}, \bar{x}_{(k+1) n}\right)$ sont dans $\Gamma_{n}$ pout tout $k$. Supposons d'abord $n=1$. L'absence de points de rebroussement et le Lemme 9 imposent qu'on ait la figure suivante:

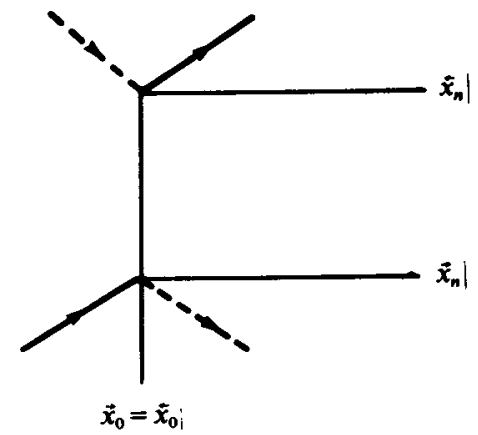

Figure 3

et il est impossible de raccorder sans violer le Lemme 9.

Dans le cas général $n>1$ on raisonne avec $\Gamma_{n}$ à la place de $\Gamma$ pour arriver à la même contradiction (les 'boîtes' $X_{k n} \times X_{(k+1) n}$ sont régulières pour $\Gamma_{n}(k>0)$ et tout point périodique de $\Gamma_{n}$ est un point périodique de $\Gamma$ ).

\section{Démonstration du Théorème 1}

Pour démontrer le Théorème 1 il suffit de montrer que, pour toute suite $\left(X_{k}\right)_{k}$, telle que ci-dessus et où aucun des $X_{k}$ n'est réduit à un point Lemme 10), toutes les boîtes $X_{k} \times X_{k+1}$ sont régulières. En effet dans ce cas le Lemme 7 assure que $\Gamma$ est le graphe d'un homéomorphisme si l'on excepte ce qui se passe à l'intérieur d'un nombre fini de boîtes $X_{k}^{i} \times X_{k+1}^{i}$. Pour obtenir le graphe $F$ du contre exemple de Denjoy cherché il suffit alors de faire un peu de 'chirurgie' dans ces dernières boîtes, 
par exemple en remplaçant $\Gamma \cap\left(X_{k}^{i} \times X_{k+1}^{i}\right)$ par la 'diagonale' du 'rectangle' $X_{k}^{i} \times$ $X_{k+1}^{i}$. C'est un vrai contre exemple de Denjoy car il admet $K$ comme ensemble invariant non trivial.

Soit donc $\left(X_{k}\right)_{k}$ une suite de boîtes non réduites à un point. Quitte à décaler les indices nous supposerons $X_{k} \times X_{k+1}$ régulière pour $k<0$ et $k \geq n(n \geq 1)$.

Si $n=1$ (seule la boîte $X_{0} \times X_{1}$ peut être non régulière). Alors le Lemme 9 permet d'affirmer que $\Gamma$ ne rencontre pas le hord de la boîte $X_{0} \times X_{1}$ en dehors des coins et le fait que $\Gamma$ ne comporte pas de point de rebroussement impose à cette boîte d'être régulière.

Si $n>1$. On raisonne alors avec $\Gamma_{n}$ à la place de $\Gamma: X_{k n} \times X_{(k+1) n}(k \in \mathbb{Z})$ devient une suite de 'boites' pour $\Gamma_{n}$ qui sont régulières pour $k \neq 0$. Par le même raisonnement que plus haut alors $X_{0} \times X_{n}$ est encore régulière pour $\Gamma_{n}$ (un point périodique de $\Gamma_{n}$ est un point périodique de $\Gamma$ ).

Supposons $n$ pair $(n=2 p)$; alors dire que $X_{0} \times X_{n}$ est régulière revient à dire que l'on a

$$
\alpha_{1} \circ \cdots \circ \alpha_{n}\left(X_{p}^{n}\right)=X_{0}, \quad \beta_{1} \circ \cdots \circ \beta_{n}\left(X_{p}^{n}\right)=X .
$$

On en déduit en particulier (les $\beta_{i}$ sont surjectifs en restriction)

$$
\beta\left(X_{n-1}^{1}\right)=X_{n} \text {. }
$$

Alors le Lemme 9 interdit à $\Gamma$ de rencontrer les bords verticaux de $X_{n-1} \times X_{n}$ et on en déduit que $X_{n-1} \times X_{n}$ est elle aussi régulière.

Raisonnant ainsi pas à pas on se ramène au cas $n=1$.

\section{Multi-applications différentiables de $S^{1}$}

Nous démontrons ici le Théorème 2 .

Soit donc une multi-application $\Gamma$ vérifiant les hypothèses de ce théorème. Le seul cas non trivial est le cas où $\alpha$ et $\beta$ ont le même degré 1 ou -1 . Quitte à reparamétrer on peut supposer que ce degré commun est 1 et alors $\Gamma$ est une multi-application monotone par morceaux. Si $\Gamma$ est un homéomorphisme c'est forcément un difféomorphisme et, ou bien il est conjugué à une rotation irrationnelle (car il vérifie les hypothèses du théorème de Denjoy) ou bien il a des orbites périodiques. Supposons que $\Gamma$ ne soit pas un homéomorphisme et n'ait pas de cycles; on peut lui appliquer le Théorème 1. On fait encore un peu de 'chirurgie' sur $\Gamma$ : on remplace tous les $\Gamma_{i j}$ qui ne sont pas des portions de difféomorphismes (ils sont en nombre fini) par une courbe $\Gamma_{i j}^{\prime}$ qui est le graphe d'un difféomorphisme de classe $C^{2}$ de $I_{i, j}$ dans $I_{i, j+1}$, de façon que la nouvelle courbe $\Gamma^{\prime}$ ainsi obtenue soit le graphe d'un difféomorphisme de classe $C^{1}$ à dérivée à variation bornée. Alors le $\Gamma^{\prime}$ ainsi obtenu serait le graphe d'un contre exemple de Denjoy de classe $C^{1}$ à dérivée à variation bornée. Ceci est impossible et achève donc la démonstration du Théorème 2.

TheOREMe 3. Soit $\Gamma$ une multi-application de $S^{1}$ admettant un paramètrage $t \rightarrow(\alpha(t), \beta(t)): S^{1} \rightarrow S^{1} \times S^{1}$ de classe $C^{\infty}$. On suppose que les relevées $\bar{\alpha}$ et $\bar{\beta} n$ 'ont pas de points où toutes les dérivées s'annulent et que $\bar{\alpha}$ ne possède pas de point 
d'inflexion à tangente horizontale. Alors ou bien $\Gamma$ est un homéomorphisme conjugué $\grave{a}$ une rotation irrationnelle ou bien $\Gamma$ admet des cycles.

La démonstration de ce théorème se conduit comme celle du Théorème 2: si $\Gamma$ n'est pas un homéomorphisme et n'a pas de cycles, on modifie son graphe comme dans la démonstration du Théorème 2. Cependant il se peut ici qu'une extrémité de courbe $\Gamma_{i j}$ soit un point à 'tangente horizontale'; on modifie alors les courbes $\Gamma_{i j}$ en des courbes $\Gamma_{i j}^{\prime}$ qui sont des graphes d'homéomorphismes de classe $C^{\infty}$ de $I_{i, j}$ dans $I_{i, j+1}$ sans points 'plats' (où toutes les dérivées sont nulles). On obtient ainsi une courbe $\Gamma^{\prime}$, modifiée de la courbe $\Gamma$, qui est le graphe d'un homéomorphisme de classe $C^{\infty}$ de $S^{1}$ dans $S^{1}$ sans points plats. Or, d'après les résultats de J. C. Yoccoz dans la référence [11], un tel homéomorphisme ne peut être un contre exemple de Denjoy. On arrive ainsi à nos fins.

\section{REFERENCES}

[1] V. I. Arnol'd. Small denominators I. A.M.S. Transl. 2(46) 213-284.

[2] J. Auslander \& Y. Katznelson. Continuous maps of the circle without periodic points. Israel $J$. Math. 32(4) (1979), 375-381.

[3] R. Bamon, I. P. Malta, M. J. Pacifico \& F. Takens. Rotation intervals of endomorphisms of the circle. Ergod. Th. \& Dynam. Sys. 4 (1984), 493-498.

[4] L. Block \& J. Franke. Existence of periodic points for maps of $S^{1}$. Inventiones Math. 22 (1973), 69-73.

[5] J. P. Dufour. Dynamique de couples de fonctions. Preprint du Séminaire de Géométrie Différentielle de Montpellier (1985-1986) France.

[6] J. P. Dufour. Stabilité simultanée de deux fonctions différentiables. Ann. Inst. Fourier, Grenoble 29(1) (1979), 263-282.

[7] J. P. Dufour. Intervalles de rotation pour les multi-applications du cercle. Comptes rendus du Colloque Géométrie Symplectique et Physique Mathématique (1986) (Lyon), à paraître.

[8] F. Hofbauer. Periodic points for piecewise monotonic transformations. Ergod. Th. \& Dynam. Sys. 5 (1985), 237-256.

[9] M. Misiurewicz. Rotation intervals for a class of maps of the real line into itself. Ergod. Th. \& Dynam. Sys. 6 (1986), 117-132.

[10] F. Przytycki. Chaos after bifurcation of a Morse-Smale diffeomorphism through a one-cycle saddle-node and iterations of multivalued mappings of an interval and a circle. Preprint 347 (85) Inst. of Math., Polish Acad. of Science.

[11] J. C. Yoccoz. Il n'y a pas de contre-exemple de Denjoy analytique. C.R. Acad. Sc. Paris, t.298, Série I, 7 (1984), 141-144. 